\title{
Structural correlation to spontaneous electric and magnetic order in multiferroic $\mathrm{LiCr}_{0.99} \mathrm{Fe}_{0.01} \mathrm{O}_{2}$
}

\author{
K. Dey, A. Karmakar, S. Majumdar, and S. Giri* \\ Department of Solid State Physics, Indian Association for the Cultivation of Science, Jadavpur, Kolkata 700 032, India
}

\begin{abstract}
We report the effect of $\mathrm{Fe}$ doping on structural, magnetic, and electric polarization in $\mathrm{LiCr}_{0.99} \mathrm{Fe}_{0.01} \mathrm{O}_{2}$. Although antiferroelectric transition remains at $62 \mathrm{~K}$, Néel temperature shifts toward higher temperature to $95 \mathrm{~K}$. This may indicate release of magnetic frustration in the antiferromagnetically coupled 2-D triangular-lattice due to minor Fe doping. Synchrotron x-ray diffraction studies reveal step-like structural transitions close to antiferroelectric and antiferromagnetic transitions. Appearance of a new structural transition at antiferromagnetic ordering indicates the presence of magnetoelastic coupling.
\end{abstract}

PACS numbers: $75.80 .+\mathrm{q}, 77.80 .-\mathrm{e}$

Multiferroics are formally defined as chemically single phase materials which possess more than one simultaneous long range ferroic order. In particular, concomitant appearance of spontaneous electric and magnetic polarizations is indicative of magnetoelectric coupling and is attractive for numerous technological applications ${ }^{1-4}$. The primary importance of these materials is the design of new devices, that can exploit their remarkable feature of controlling magnetic or dielectric properties by the application of an electric or magnetic field, respectively. Multiferroicity usually involves breaking of inversion symmetry during ferro(antiferro)electric order and is usually associated with first order structural change. Thus elastic coupling is intimately correlated to spontaneous electric and/or magnetic ordering of multiferroic materials ${ }^{5-9}$.

The crystal structure of $\mathrm{LiCrO}_{2}$ (LCO) possesses 2dimensional (2D) triangular lattice formed by $\mathrm{Cr}$ sublattices which are antiferromagnetically coupled ${ }^{10-15}$. In compatibility with $2 \mathrm{D}$ structure, intralayer superexchange interaction is significantly stronger than interlayer exchange interaction ${ }^{13}$. Below antiferromagnetic (AFM) ordering $\left(T_{N}=62 \mathrm{~K}\right)$, the double-Q $120^{\circ}$ spin structure with nonequivalent wave numbers is characterized by alternate stacking of $\mathrm{Cr}^{3+}$ layers with opposite vector spin chirality along the crystallographic $c$ axis $^{16,17}$. The proper screw-type magnetic structure with opposite spin chirality in the alternative $\mathrm{Cr}$ layers provide antiferroelectric (AFE) ordering at $T_{N}{ }^{18}$. Our recent study on the nominal doping of $\mathrm{Cu}$ in $\mathrm{Li}_{0.99} \mathrm{Cu}_{0.01} \mathrm{CrO}_{2}$ (LCCO) significantly enhanced the interlayer coupling and led to strong magnetoelastic effect with an unconventional nature of electric polarization at $T_{N}{ }^{19}$. In the present work we have done synchrotron X-ray diffraction study of $\mathrm{LiCr}_{0.99} \mathrm{Fe}_{0.01} \mathrm{O}_{2}$ (LCFO) over a wide temperature range, 20-300 K. We observe that minor Fe doping in the $\mathrm{Cr}$ network remarkably changes structural properties. Beside the step-like change at $62 \mathrm{~K}$, as also evident in un-

*Electronic address: sspsg2@iacs.res.in doped LCO, another new step-like change is observed in $\mathrm{Cr}-\mathrm{O}$ bond length and $\mathrm{O}-\mathrm{Cr}-\mathrm{O}$ bond angle at $95 \mathrm{~K}$. Although AFE ordering remains at $62 \mathrm{~K}, T_{N}$ shifts toward higher temperature to $95 \mathrm{~K}$ compared to undoped LCO. This indicates release of magnetic frustration due to nominal Fe doping. Importantly, the step-like changes in $\mathrm{Cr}-\mathrm{O}$ bond length and $\mathrm{O}-\mathrm{Cr}-\mathrm{O}$ bond angle are observed close to $T_{N}$ pointing out the magnetoelastic coupling. The results of doping in the Cr network are distinct from that of nonmagnetic Li site doping ${ }^{19}$. The different results of LCFO from LCCO are argued in the context of different structural changes at antiferroelectric and antiferromagnetic transition temperatures in the $2 \mathrm{D}$ ordered rocksalt structure.

Polycrystalline $\mathrm{LiCr}_{0.99} \mathrm{Fe}_{0.01} \mathrm{O}_{2}$ is prepared by solid state reaction described elsewhere ${ }^{19}$. Chemical composition is confirmed using X-ray diffraction studies at room temperature recorded in a SEIFERT X-ray diffractometer (Model: XRAY3000P) using $\mathrm{Cu} \mathrm{K}_{\alpha}$ radiation and is further verified by synchrotron X-ray powder diffraction studies measured at P07 beam line of Petra III, Hamburg, Germany at a wavelength of $0.1347 \AA \quad(92 \mathrm{keV})$. The diffraction data are refined through Rietveld technique using a commercial MAUD (materials analysis using diffraction) software. Dielectric properties are measured in an E4980A LCR meter (Agilent Technologies, USA) equipped with a cryogen free cryocooler (JANIS, USA). The pyroelectric current $\left(I_{p}\right)$ is recorded in an electrometer (Keithley, model 6517B) at a constant temperature sweep rate $(4.0 \mathrm{~K} / \mathrm{min})$ and integrated with time for obtaining thermal variation of electric polarization $(P)$. Electrical contacts are prepared using an air drying silver paint. The dc magnetization is recorded in a commercial magnetometer of Quantum Design (MPMS, evercool).

Thermal variation of zero-field cooled (ZFC) and fieldcooled (FC) magnetization are shown in Figs. 1a and $1 \mathrm{~b}$ measured at 10 and $100 \mathrm{Oe}$, respectively. The ZFC magnetization is strongly influenced by the measuring field. In case of measurement at 10 Oe, a peak is observed close to $95 \mathrm{~K}$ along with a shoulder around $62 \mathrm{~K}$, at which both AFM and AFE orders are observed for undoped LCO. The results reveal that $T_{N}$ shifts toward 


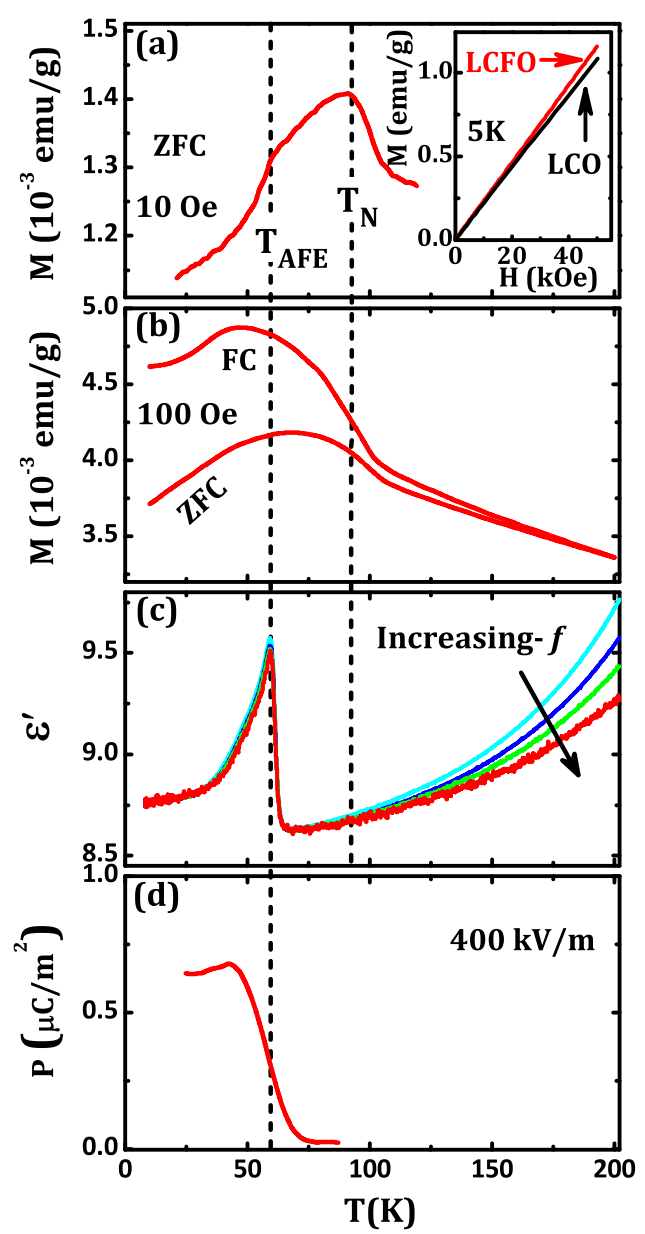

FIG. 1: (Color online) Thermal variation of ZFC magnetization measured at 10 Oe (a) and ZFC-FC magnetization measured at 100 Oe (b). Inset of (a) displays linear magnetization curves for LCFO and LCO at $5 \mathrm{~K}$. Thermal variation of real component of dielectric constant, $\epsilon^{\prime}$ (c) and electric polarization, $P(\mathrm{~d})$.

higher temperature compared to the value for LCO. The small doping of Fe in the Cr network, which forms antiferromagnetically coupled 2D triangular lattice, releases magnetic frustration, as indicated by the increase of $T_{N}$. As displayed in Fig. 1b the ZFC-FC magnetization overlaps above $200 \mathrm{~K}$ which is much higher than $T_{N}$ at 95 $\mathrm{K}$. At $5 \mathrm{~K}$ linearity of the magnetization curves for LCO and LCFO are indicative of AFM ordering. The linearity of the magnetization curve of LCFO is contrary to the observed significant coercivity for $\mathrm{LCCO}^{19}$. This appears due to different structural changes in the two compounds as discussed elsewhere below.

The real component of dielectric permittivity $\left(\epsilon^{\prime}\right)$ recorded at different frequencies $(f)$ is displayed in Fig. 1c. The $\epsilon^{\prime}(T)$ exhibits a sharp peak at $62 \mathrm{~K}$ and it does not show any convincing change in $\epsilon^{\prime}$ around $T_{N}$. The peak at $62 \mathrm{~K}$ does not show any frequency dependence,
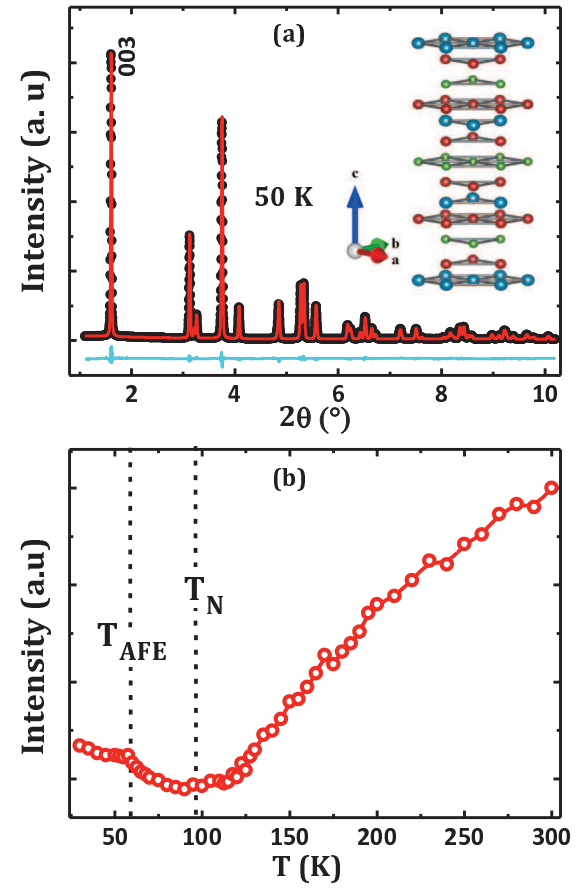

FIG. 2: (Color online) (a) Rietveld refinement of X-ray powder diffraction patterns (black symbols) at $50 \mathrm{~K}$ for LCFO. Solid curve demonstrates the fit. The plot at the bottom is the residual. Inset shows layered arrangements of the atomic positions as obtained from the refined values. (b) Thermal variation of the intensities of (003) peak.

which indicates long-range order of electric dipole moments $\left(T_{A F E}\right)$. This is further confirmed by polarization $(P)$ measurement as displayed in Fig. 1d. The $P$ is calculated by integrating pyroelectric current recorded in an electrometer at a poling field $400 \mathrm{kV} / \mathrm{m}$. Magnitude and nature of the thermal variation below $T_{A F E}$ indicate AFE order similar to that proposed for undoped $\mathrm{LCO}^{18}$.

The structural properties are thoroughly studied by X-ray powder diffraction studies using a high-flux synchrotron source over $20-300 \mathrm{~K}$ for LCFO. The structural parameters are obtained from careful analyses of the diffraction patterns using Rietveld refinement procedure. The refinement is performed using $R \overline{3} \mathrm{~m}$ space group with atomic positions $\operatorname{Li}\left(\begin{array}{lll}0 & 0 & 1 / 2\end{array}\right), \mathrm{Cr}\left(\begin{array}{lll}0 & 0 & 0\end{array}\right)$, and $\mathrm{O}(00 \mathrm{z})$. The diffraction patterns in the entire temperature range can be fitted with the same $R \overline{3} m$ space group. One of the representative examples of the fit (continuous curve) with the experimental data (symbols) is shown in Fig. 2 a at $50 \mathrm{~K}$, which is below $T_{A F E}$ as well as $T_{N}$. The difference plot is shown at the bottom indicating the absence of any secondary phase. Reasonably small values of the reliability parameters, $R_{w}(\sim 3.64)$, $R_{\text {exp }}(\sim 3.29)$, and $\chi^{2}(\sim 1.03)$ assure the authenticity of the refinement. Inset of Fig. 2a shows the layered arrangements of the atomic positions as obtained from the Rietveld refinement. Thermal variation of the intensity 

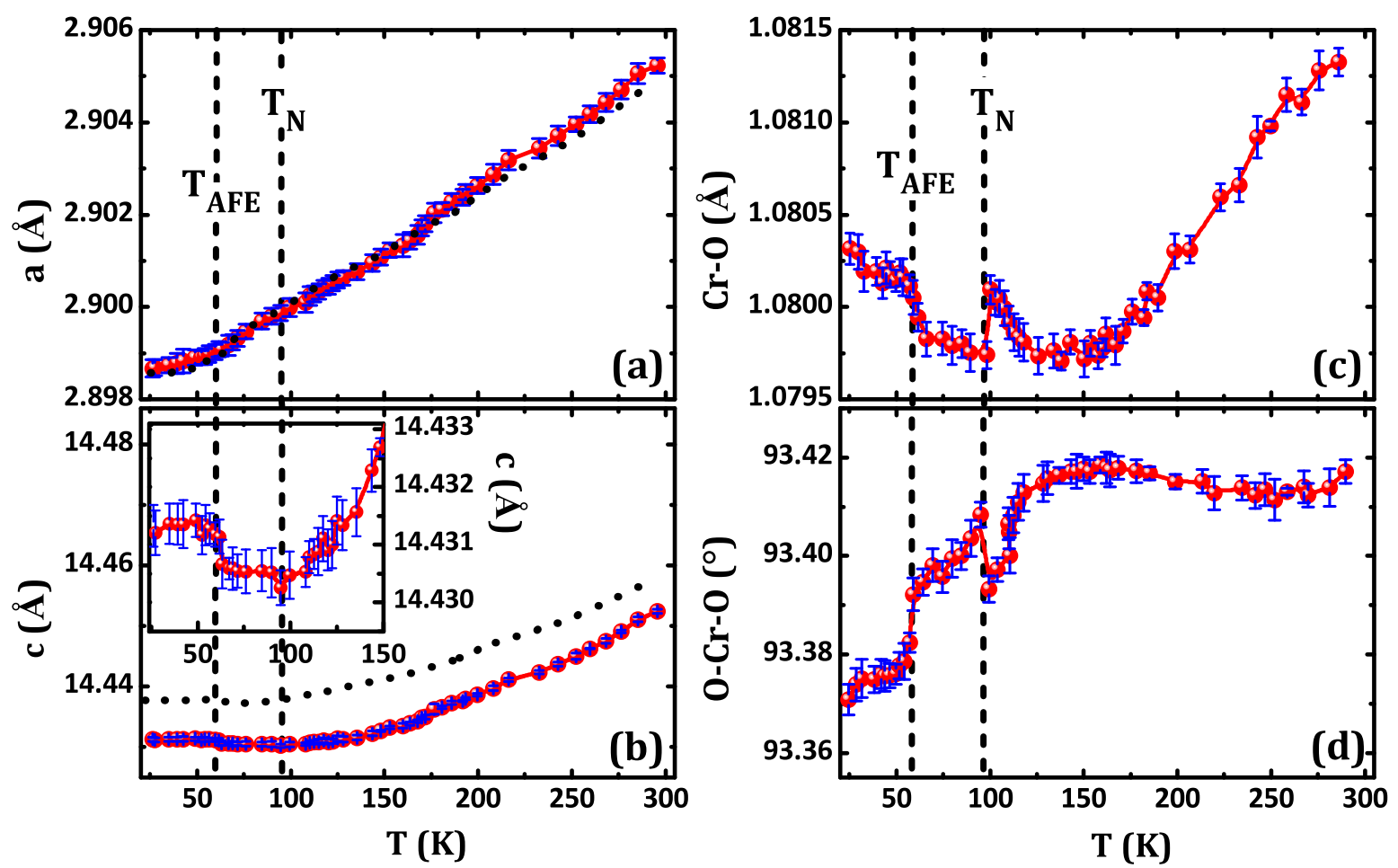

FIG. 3: (Color online) Thermal variations of $a$ (a), $c$ (b), $\mathrm{Cr}-\mathrm{O}$ bond length (c), and $\mathrm{O}-\mathrm{Cr}-\mathrm{O}$ bond angle (d) for LCFO. Broken curves shown in figures (a) and (b) displaying thermal variations for undoped LCO as obtained from our previous report ${ }^{19}$.

of (003) peak is displayed in Fig. 2b, which shows a minimum around $95 \mathrm{~K}$, below which it increases slowly followed by an anomaly at $62 \mathrm{~K}$. These are the characteristic temperatures at which $T_{N}$ and $T_{A F E}$ are observed. The signature of $T_{A F E}$ in the peak intensity is consistent with the observed anomaly or sharp change of $T$ variation in the integrated intensity at the ferroelectric transition as found in the literature ${ }^{20-22}$

Temperature variation of parameters $a$ and $c$ are shown in Fig. 3a and 3b, respectively. Thermal variations of these parameters are compared with that obtained (broken curves in Figs. 3a and 3b) from our previous reports for $\mathrm{LCO}^{19}$. a decreases with decreasing temperature while $c$ decreases with decreasing temperature until $\sim 95 \mathrm{~K}$, below which slow increase is observed followed by a step like rise at $62 \mathrm{~K}$ as evident in the inset of Fig. 3b. Thermal variation of $a$ is similar to that observed in $\mathrm{LCO}$ and $\mathrm{LCCO}^{19}$. In fact, $a$ of LCO almost overlaps with the result for LCFO as shown in Fig. 3a. On the other hand, parameter $c$ decreases significantly in the measured temperature range compared to LCO, which is analogous to $\mathrm{LCCO}^{19}$. This indicates that minor doping even at the $\mathrm{Cr}$ site significantly reduces the interlayer distance. The step like increase in $c$ at $62 \mathrm{~K}$ was also observed for LCO and LCCO. Signatures of $T_{N}$ and $T_{A F E}$ are more evident in the thermal variations of $\mathrm{Cr}-\mathrm{O}$ bond length $\left(d_{C r-O}\right)$ and $\mathrm{O}-\mathrm{Cr}-\mathrm{O}$ bond angles $(\alpha)$ as shown in Figs. 3c and 3d, respectively. In consistency with that observed in $c(T)$, a more apparent step like rise in $d_{C r-O}$ and fall in $\alpha$ are observed at $62 \mathrm{~K}$. This sharp change is $\sim 2$ times larger than that observed for undoped LCO. It is, however, nearly half in magnitude at the step-like change observed in $\mathrm{LCCO}^{19}$. In addition to this sharp change at $T_{A F E}$, another new transition is evident at $95 \mathrm{~K}$ which is absent in undoped $\mathrm{LCO}^{19}$. Interestingly, this new step like transition in $d_{C r-O}$ and $\alpha$ are observed close to $T_{N}$ revealing strong magnetoelastic coupling. Similar magnetoelastic coupling was also observed in LCCO, although magnitude of step-like change at $T_{N}$ was $\sim 4$ times enhanced and $T_{N}$ was shifted to more higher temperature $\sim 118 \mathrm{~K}^{19}$.

Current investigation infers that doping at the Cr site significantly enhances magnetoelastic coupling compared to undoped LCO. This is, however, analogous to that observed in $\mathrm{Cu}$ doped LCCO, although magnetic and dielectric properties are significantly different. Unusual ferrielectric-type order with a significant uncompensated polarization and ferrimagnetic-type magnetic ordering with a large coercivity are observed for LCCO whereas antiferroelectric and antiferromagnetic ordering is proposed for LCFO in the current investigation, analogous to that observed in LCO. Markedly different electric and magnetic polarizations in LCFO and LCCO are probably related to the magnitude of step-like changes at $T_{N}$ and 
$T_{A F E}$ which are $\sim 4$ and $\sim 2$ times enhanced in LCCO than LCFO. Current investigation further confirms that doping either at nonmagnetic Li site or magnetic $\mathrm{Cr}$ site decouples electric and magnetic order. The Fe doping in LCFO directly creates a disorder in the 2D triangular lattice formed by $\mathrm{Cr}$ sublattice. This leads to the key effect of releasing the frustration in the antiferromagnetically coupled $2 \mathrm{D}$ triangular lattice and causes increase of $T_{N}$ for LCFO.

In summary, Fe doping in $\mathrm{LiCr}_{0.99} \mathrm{Fe}_{0.01} \mathrm{O}_{2}$ strongly influences magnetic and structural properties, although dielectric properties and antiferroelectric ordering temperature do not alter significantly. Antiferromagnetic Néel temperature shifts toward higher temperature as a result of Fe doping. Careful Rietveld refinement of the synchrotron X-ray diffraction patterns over wide temperature range provides thermal variations of lattice constants and microscopic structural parameters. Thermal variations of $\mathrm{Cr}-\mathrm{O}$ bond length and $\mathrm{O}-\mathrm{Cr}-\mathrm{O}$ bond angles demonstrate step-like transitions at $T_{A F E}$ and $T_{N}$. The step-like transition at $T_{N}$ is a new observation and appears due to Fe doping revealing a magnetoelastic coupling.

\section{Acknowledgment}

S.G. acknowledges financial support for the X-ray diffraction studies using synchrotron radiation under the DSTDESY project. S.G. also sincerely acknowledges Uta Rütt and O. Gutowski of HASYLAB, DESY, Germany, for recording the X-ray synchrotron data and wishes to thank Council for Scientific and Industrial Research (CSIR), India for the financial support under grand No. 03(1167)/10/EMR-II and also DST Unit of Nanoscience at IACS for providing MPMS measurement facility. K.D. acknowledges CSIR for the senior research fellowship.
[1] Fiebig M. J. Phys. D: Appl. Phys. 2005;38:R123.

[2] Cheong S-W, Mostovoy M. Nat. Mater. 2007;6:13.

[3] Khomskii D. I. J. Magn. Magn. Mat. 2006;306:1.

[4] Eerenstein W, Mathur ND, Scott J. F. Nature 2006;442:759.

[5] Khomchenkoa VA, Shvartsman VV, Borisov P, Kleemann W, Kiselev DA, Bdikin IK, Vieira JM, Kholkin A.L. Acta Mater. 2009;57:5137.

[6] Khomchenko VA, Paixãa JA, Shvartsman VV, Borisov P, Kleemann W, Karpinsky DV, Kholkin A.L. Scripta Mater. 2010;62:238.

[7] Singh AK, Patnaik S, Kaushik SD, Siruguri V Phys. Rev. B 2010;81:184406.

[8] Dey K, Majumdar S, Giri S. Acta Mater. 2013;61:379.

[9] Haumont R, Kornev I A, Lisenkov S, Bellaiche L, Kreisel J, Dkhil B. Phys. Rev. B 2008;78:134108.

[10] Hemmida M, Krug von Nidda H-A, Büttgen N, Loidl A, Alexander LK, Nath R, Mahajan AV, Berger RF, Cava RJ, Singh Y, Johnston D.C. Phys. Rev. B 2009;80:054406.

[11] Mazin I.I. Phys. Rev. B 2007;75:094407.

[12] Moreno NO, Israel C, Pagliuso PG, Garcia-Adevac AJ, Rettori C, Sarrao JL, Thompson JD, Oseroff S.B. J. Magn. Magn. Mater. 2004;272-276:e1023.

[13] Alexander LK, Büttgen N, Nath R, Mahajan AV, Loidl
A. Phys. Rev. B 2007;76:064429.

[14] Sugiyama J, Mansson M, Ikedo Y, Goko T, Mukai K, Andreica D, Amato A, Ariyoshi K, Ohzuku T. Phys. Rev. B 2009;79:184411.

[15] Olariu A, Mendels P, Bert F, Alexander LK, Mahajan AV, Hillier AD, Amato A. Phys. Rev. B 2009;79:224401.

[16] Soubeyroux JL, Fruchart D, Marmeggi JC, Fitzgerald WJ, Delmas C, Le Flem G. Phys. Stat. Solidi 1981;67:633.

[17] Kadowakit H, Takeit H, Motoya K.J. Phys.: Condens. Matter 1995;7:6869.

[18] Seki S, Onose Y, Tokura Y. Phys. Rev. Lett. 2008;101:067204.

[19] Dey K, Karmakar A, Majumdar S, Giri S. Phys. Rev. B 2013;87:094403.

[20] Koo J, Song C, Ji S, Lee J-S, Park J, Jang T-H, Yang C-H, Park J-H, Jeong YH, Lee K-B, Koo TY, Park YJ, Kim J-Y, Wermeille D, Goldman AI, Srajer G, Park S, Cheong S.-W Phys. Rev. Lett. 2007;99:197601.

[21] Horiuchi S, Kumai R, Okimoto Y, Tokura Y. Phys. Rev. Lett. 2000;85:5210.

[22] Fong DD, Stephenson GB, Streiffer SK, Eastman JA, Auciello O, Fuoss PH, Thompson C. Science 2004;304:1650. 